Commun. Fac. Sci. Univ. Ank. Ser. A1 Math. Stat.

Volume 69, Number 1, Pages 794-814(2020)

DOI: $10.31801 /$ cfsuasmas. 597680

ISSN 1303-5991 E-ISSN 2618-6470

http://communications.science.ankara.edu.tr/index.php?series=A1

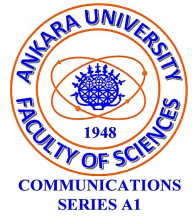

\title{
THE COMPARISON OF DIFFERENT ESTIMATION METHODS FOR THE PARAMETERS OF FLEXIBLE WEIBULL DISTRIBUTION
}

\author{
SAJID ALI, SANKU DEY, M. H. TAHIR, AND MUHAMMAD MANSOOR
}

\begin{abstract}
This article presents different parameter estimation methods for flexible Weibull distribution introduced by Bebbington et al. (Reliability Engineering and System Safety 92:719-726, 2007), which is a modified version of the Weibull distribution and is suitable to model different shapes of the hazard rate. We consider both frequentist and Bayesian estimation methods and present a comprehensive comparison of them. For frequentist estimation, we consider the maximum likelihood estimators, least squares estimators, weighted least squares estimators, percentile estimators, the maximum product spacing estimators, the minimum spacing absolute distance estimators, the minimum spacing absolute log-distance estimators, Cramér von Mises estimators, Anderson Darling estimators, and right tailed Anderson Darling estimators, and compare them using a comprehensive simulation study. We also consider Bayesian estimation by assuming gamma priors for both shape and scale parameters. We use a Markov Chain Monte Carlo algorithm to compute the posterior summaries. A real data example is also a part of this work.
\end{abstract}

\section{INTRODUCTION}

Weibull distribution is one of the most widely used distributions in reliability, and has a monotonic hazard rate, which may be increasing or decreasing. In many reliability applications, however, the failure rate often non-monotonic, which motivated [1 to introduce a new extension of the Weibull distribution having bathtub-shaped failure rate. To define it, let $X$ have the flexible Weibull (FW for short) distribution, say $X \sim \mathrm{FW}(\alpha, \lambda)$. [1] defined the cumulative distribution function (cdf) of

Received by the editors: July 28, 2019; Accepted: March 04, 2020.

2010 Mathematics Subject Classification. Primary 62E10; Secondary 62F99, 60E05.

Key words and phrases. Weibull distribution; maximum likelihood estimators, least squares estimators, weighted least squares estimators, percentile estimators, maximum product spacing estimators, minimum spacing absolute distance estimators, minimum spacing absolute log-distance estimators, Cramér von Mises estimators, Anderson Darling estimators, right tailed Anderson Darling estimators, Bayesian estimation.

(C) 2020 Ankara University Communications Faculty of Sciences University of Ankara-Series A1 Mathematics and Statistics 
$X$ as

$$
G(x)=1-\exp (-\exp (\alpha t-\lambda / t)),
$$

where $\alpha$ and $\lambda$ are the shape parameters. The exponential distribution is obtained by $\lambda=0$ and $\alpha=\log (\theta)$. The probability density function (pdf) corresponding to (1) is given by

$$
g(x)=\left(\alpha+\lambda / x^{2}\right) \exp (\alpha t-\lambda / x) \exp (-\exp (\alpha t-\lambda / t)), \quad x>0
$$

[1] pointed out that as $\lambda$ decreases, the failure rate function becomes more bathtub-like while it becomes shallower as $\alpha$ increases.

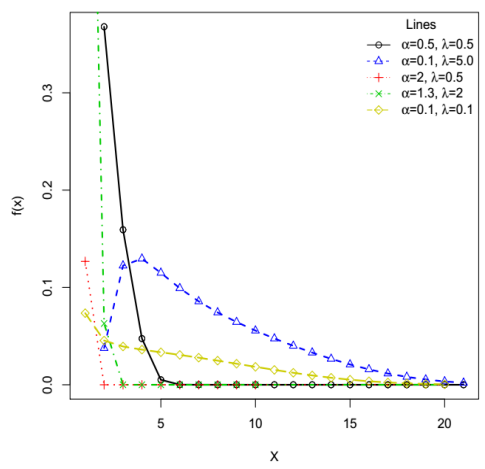

FiguRE 1. Density Plot of flexible Weibull for some selected parameter values.

Note that the FW distribution has the closed-form density, hazard and survival functions. In Figure-1, we have depicted the density of FW distribution for various combinations of parameters. It is clear from the figure that the distribution is very flexible and adopts various shapes for different combinations of parameters.

In the literature, [2] developed a $\mathrm{R}$ Package 'reliaR' to generate random numbers from FW to estimate its parameters and study other reliability characteristics. [3] discussed Bayesian estimation and prediction for FW under type-II censoring scheme. 44 discussed parameter estimation of the flexible Weibull distribution for type I censored data. [5] proposed a new extension of FW distribution using the odd generalized exponential generator. 6] proposed a generalized class of FW distribution for repairable systems. [7 proposed a generalized class of $\mathrm{FW}$ distribution. [8] discussed estimation and prediction for type-II hybrid censored data assuming FW distribution. 9 studied the penalized maximum likelihood estimation for the modified extended Weibull distribution. [10] discussed the reliability properties of the proportional hazard reverse transformation using $\mathrm{FW}$ distribution. 11] presented estimation and prediction for FW based on progressive type-II censored data. 12 . proposed exponentiated additive Weibull distribution where $\mathrm{FW}$ is a special case of the proposed distribution. 
The aim of this article is to compare different parameter estimation methods, including both classical and Bayesian. In particular, we compare the maximum likelihood, the maximum and the minimum spacing distances (minimum spacing absolute distance and minimum spacing absolute-log distance), ordinary and weighted least squares, percentiles, the minimum distance methods including Cramér-vonMises, Anderson-Darling and right-tail Anderson-Darling. Further, we also compute the parameter estimates of FW by using the Bayesian method, where we use the Markov Chain Monte Carlo (MCMC) to obtain the posterior summaries. Several authors have used different methods of estimations for different distributions, for example, [13, 14, 15, [16, 17, 18, 19, 20].

The rest of the article is organized as follows: Section 2 discusses some new properties of the FW distribution. Section 3 deals with different methods of estimation of the model parameters. Section 4 presents simulation study while a real life example to show the practical application is presented in Section 5. Finally, some concluding remarks are given in Section 6 .

\section{NEW PROPERTIES}

This section discusses some statistical properties.

2.1. Moments, Skewness and Kurtosis. We calculate the mean, variance, skewness and kurtosis numerically and depict in Figure-2. It is clear from the figure that as $\lambda$ increases, the mean and variance also increase. However, the skewness and kurtosis decrease by increasing $\lambda$. It is also noticed that a small value of $\alpha$ results into large value of mean, variance, skewness and kurtosis.

2.2. Quantile function. To generate random variable from FW, we invert Equation1 as follows $X=F^{-1}(u)$, where $u \sim U$ niform $(0,1)$. The simplified form is

$$
X=F^{-1}(u)=\frac{1}{2 \alpha}\left(\log (-\log u)+\sqrt{\{\log (-\log u)\}^{2}+4 \alpha \lambda}\right)
$$

The skewness and kurtosis measures can be investigated using the quantile function. For example, the Bowley skewness [21] based on quantiles is given by

$$
B=\frac{F^{-1}(3 / 4)+F^{-1}(1 / 4)-2 F^{-1}(2 / 4)}{F^{-1}(3 / 4)-F^{-1}(1 / 4)} .
$$

Similarly, the Moors' kurtosis [22] is

$$
M=\frac{F^{-1}(3 / 8)-F^{-1}(1 / 8)+F^{-1}(7 / 8)-F^{-1}(5 / 8)}{F^{-1}(6 / 8)-F^{-1}(2 / 8)} .
$$

2.3. Reliability properties of FW distribution. A key property to characterize the distribution is log-concave, i.e., the density is log-concave if $d^{2} / d x^{2} \log f<0$, otherwise convex. The hazard would be decreasing if density is log-concave. For the FW, it is observed that the density is log-concave for $\lambda>\alpha$. 


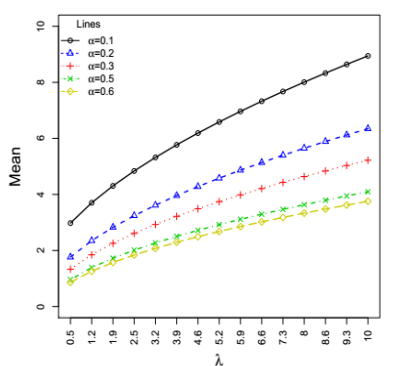

(a)

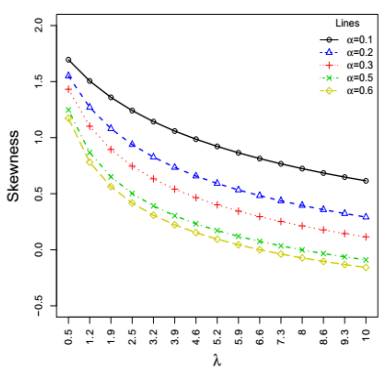

(c)

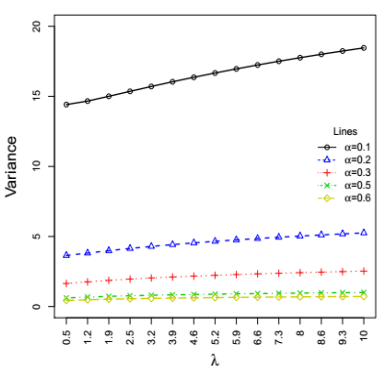

(b)

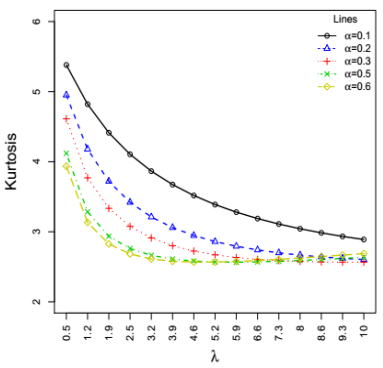

(d)

Figure 2. Plots of the FW (a) Mean (b) Variance (c) Skewness, and (d) Kurtosis for some selected parameter values.

2.4. Stochastic ordering. Stochastic ordering is an important tool in reliability theory and finance to assess comparative behavior. Let $X_{1}$ and $X_{2}$ be two random variables having cdfs, sfs and pdfs $F_{1}(x), F_{2}(x), \bar{F}_{1}(x)=1-F_{1}(x)$, $\bar{F}_{2}(x)=1-F_{2}(x), f_{1}(x)$, and $f_{2}(x)$, respectively. The random variable $X_{1}$ is said to be smaller than $X_{2}$ in the following ordering as:

(i) stochastic order (denoted by $X_{1} \leq s t X_{2}$ ) if $\bar{F}_{1}(x) \leq \bar{F}_{2}(x)$ for all $x$;

(ii) likelihood ratio order (denoted by $X_{1} \leq_{l r} X_{1}$ ) if $f_{1}(x) / f_{2}(x)$ is decreasing in $x \geq 0$;

(iii) hazard rate order (denoted by $X_{1} \leq_{h r} X_{2}$ ) if $\bar{F}_{1}(x) / \bar{F}_{2}(x)$ is decreasing in $x \geq 0$;

(iv) reversed hazard rate order (denoted by $X_{1} \leq_{r h r} X_{2}$ ) if $F_{1}(x) / F_{2}(x)$ is decreasing in $x \geq 0$.

All these four stochastic orders defined in (i)-(iv) are related to each other [23] and the following implications hold:

$$
\left(X_{1} \leq_{r h r} X_{2}\right) \Leftarrow\left(X_{1} \leq_{l r} X_{2}\right) \Rightarrow\left(X_{1} \leq_{h r} X_{2}\right) \Rightarrow\left(X_{1} \leq_{s t} X_{2}\right) .
$$


The following theorem shows that the FW distribution has likelihood ratio ordering when appropriate assumptions are satisfied.

Theorem 2.1. Let $X_{1} \sim F W\left(\alpha_{1}, \lambda_{1}\right)$ and $X_{2} \sim F W\left(\alpha_{2}, \lambda_{2}\right)$. If $\alpha_{1}<\alpha_{2}$ for fixed $\lambda_{1}=\lambda_{2}=\lambda$, and $\lambda_{2}>\lambda_{2}$, for $\alpha_{1}=\alpha_{2}=\alpha$ then $X_{1} \leq_{l r} X_{2}$.

Proof. It is not difficult to show that $\frac{d}{d x} \log \frac{f_{1}\left(x ; \alpha_{1}, \lambda_{1}\right)}{f_{2}\left(x ; \alpha_{2}, \lambda_{1}\right)}<0$ for the following conditions:

- $\alpha_{1}<\alpha_{2}$ for fixed $\lambda_{1}=\lambda_{2}=\lambda$,

- $\lambda_{2}>\lambda_{2}$ and $\alpha_{1}=\alpha_{2}=\alpha$.

Thus, likelihood ratio ordering holds and $X_{1} \leq_{l r} X_{2}$.

2.5. Stress and Strength Analysis. Stress-Strength reliability is defined as $G=$ $\operatorname{Pr}\left(X_{1}>X_{2}\right)=\int_{0}^{\infty} f_{1}(x) F_{2}(x) d x, X_{1} \sim F W\left(\alpha_{1}, \lambda_{1}\right)$ and $X_{2} \sim F W\left(\alpha_{2}, \lambda_{2}\right)$, whereas the $f_{1}(x)$ is the pdf of $X_{1}$ and $F_{2}(x)$ cdf of $X_{2}$.

$$
\begin{aligned}
G=\operatorname{Pr}\left(X_{1}>X_{2}\right) & =1-\int_{0}^{\infty}\left(\alpha_{1}+\lambda_{1} / x^{2}\right) \exp \left(\alpha_{1} x-\lambda_{1} / x\right) \\
\times & \exp \left(-\exp \left(\alpha_{1} x-\lambda_{1} / x\right)-\exp \left(\alpha_{2} x-\lambda_{2} / x\right)\right) d x
\end{aligned}
$$

The above equation can be solved numerically.

\section{Parameters estimation methods}

This section describes ten different methods of estimation to obtain the estimators of the parameters $\alpha$ and $\lambda$ of the FW distribution.

3.1. Maximum likelihood estimators. Let $x_{1}, x_{2}, \ldots, x_{n}$ be a random sample of size $n$ from Equation (2). Then, the log-likelihood function is given by

$$
\begin{aligned}
\ell(\alpha, \lambda)= & \sum_{i=1}^{n} \log \left(\alpha+\lambda / x_{i}^{2}\right) \\
& +\alpha \sum_{i=1}^{n} x_{i}-\sum_{i=1}^{n}\left(\lambda / x_{i}\right)-\sum_{i=1}^{n} \exp \left(\alpha x_{i}-\lambda / x_{i}\right)
\end{aligned}
$$

The resulting partial derivatives of the log-likelihood function are

$$
\begin{gathered}
\frac{\partial \ell(\alpha, \lambda)}{\partial \alpha}=\sum_{i=1}^{n} \frac{1}{\alpha+\lambda / x_{i}^{2}}+\sum_{i=1}^{n} x_{i}-\sum_{i=1}^{n} x_{i} \exp \left(\alpha x_{i}-\lambda / x_{i}\right) \\
\frac{\partial \ell(\alpha, \lambda)}{\partial \lambda}=\sum_{i=1}^{n} \frac{1}{\alpha x_{i}^{2}+\lambda}+\sum_{i=1}^{n} x_{i}^{-1}-\sum_{i=1}^{n} x_{i}^{-1} \exp \left(\alpha x_{i}-\lambda / x_{i}\right)
\end{gathered}
$$

Equating these partial derivatives to zero do not yield closed-form solutions for the MLEs and thus a numerical method, like Newton Raphson, is used for solving these equations simultaneously. 
3.2. Least Squares Estimators. The least squares and weighted least squares estimators were proposed by [24] to estimate the parameters of beta distributions. To define these, suppose $F\left(X_{(j)}\right)$ denote the distribution function of the ordered random variables $X_{(1)}<X_{(2)}<\cdots<X_{(n)}$ where $\left\{X_{1}, X_{2}, \cdots, X_{n}\right\}$ is a random sample of size $n$ from the distribution function $F(\cdot)$. Then, the least squares estimators of $\alpha$ and $\lambda$, say $\hat{\alpha}_{L S E}$ and $\hat{\lambda}_{L S E}$ can be obtained by minimizing

$$
S(\alpha, \lambda)=\sum_{i=1}^{n}\left[F\left(x_{i: n} \mid \alpha, \lambda\right)-\frac{i}{n+1}\right]^{2}
$$

with respect to $\alpha$ and $\lambda$, where $F(\cdot)$ is the cdf (1). Equivalently, the estimators can be obtained by solving:

$$
\begin{aligned}
& \sum_{i=1}^{n}\left[F\left(x_{i: n} \mid \alpha, \lambda\right)-\frac{i}{n+1}\right] \eta_{1}\left(x_{i: n} \mid \alpha, \lambda\right)=0, \\
& \sum_{i=1}^{n}\left[F\left(x_{i: n} \mid \alpha, \lambda\right)-\frac{i}{n+1}\right] \eta_{2}\left(x_{i: n} \mid \alpha, \lambda\right)=0
\end{aligned}
$$

where

$$
\eta_{1}\left(x_{i: n} \mid \alpha, \lambda\right)=\alpha \exp ((\alpha x-\lambda / x)-\exp (\alpha x-\lambda / x))
$$

and

$$
\eta_{2}\left(x_{i: n} \mid \alpha, \lambda\right)=\frac{\lambda}{x^{2}} \exp ((\alpha x-\lambda / x)-\exp (\alpha x-\lambda / x)) .
$$

The weighted least squares estimators, $\widehat{\alpha}_{W L S E}$ and $\widehat{\lambda}_{W L S E}$, can be obtained by minimizing

$$
W(\alpha, \lambda)=\sum_{i=1}^{n} \frac{(n+1)^{2}(n+2)}{i(n-i+1)}\left[F\left(x_{i: n} \mid \alpha, \lambda\right)-\frac{i}{n+1}\right]^{2} .
$$

These estimators can be obtained by solving:

$$
\begin{aligned}
& \sum_{i=1}^{n} \frac{(n+1)^{2}(n+2)}{i(n-i+1)}\left[F\left(x_{i: n} \mid \alpha, \lambda\right)-\frac{i}{n+1}\right] \eta_{1}\left(x_{i: n} \mid \alpha, \lambda\right)=0, \\
& \sum_{i=1}^{n} \frac{(n+1)^{2}(n+2)}{i(n-i+1)}\left[F\left(x_{i: n} \mid \alpha, \lambda\right)-\frac{i}{n+1}\right] \eta_{2}\left(x_{i: n} \mid \alpha, \lambda\right)=0 .
\end{aligned}
$$

3.3. Percentile Estimators. If the data come from a distribution function which has a closed form, then the unknown parameters can be estimated by fitting straight line to the theoretical points obtained from the distribution function and the sample percentile points. This method was originally suggested by [25, 26] and it has been used for Weibull distribution and for generalized exponential distribution. In this paper, we apply the same technique for the two-parameter FW distribution. Let $X_{(j)}$ be the $j$ th order statistic, i.e, $X_{(1)}<X_{(2)}<\cdots<X_{(n)}$. If $p_{j}$ denote 
some estimate of $F\left(x_{(j)} ; \alpha, \lambda\right)$, then the estimate of $\alpha$ and $\lambda$ can be obtained by minimizing

$$
\sum_{j=1}^{n}\left(x_{(j)}-\frac{1}{2 \alpha}\left(\log \left(-\log p_{j}\right)+\sqrt{\left\{\log \left(-\log p_{j}\right)\right\}^{2}+4 \alpha \lambda}\right)\right)^{2},
$$

with respect to $\alpha$ and $\lambda$. Several type of estimators for $p_{j}$ can be used 27] and this paper considers $p_{j}=\frac{j}{n+1}$.

3.4. Maximum and Minimum Product of Spacings Estimators. The maximum product spacing (MPS) method was introduced by [28, 29] as an alternative to MLE for the estimation of the unknown parameters of continuous univariate distributions. The MPS method was also derived independently by [30] as an approximation to the Kullback-Leibler measure of information. To motivate our choice, 29] proved that this method is as efficient as the MLE estimators and consistent under more general conditions.

We define the uniform spacings of a random sample from the FW distribution as:

$$
D_{i}(\alpha, \lambda)=F\left(x_{i: n} \mid \alpha, \lambda\right)-F\left(x_{i-1: n} \mid \alpha, \lambda\right), \quad i=1,2, \ldots, n,
$$

where $F\left(x_{0: n} \mid \alpha, \lambda\right)=0$ and $F\left(x_{n+1: n} \mid \alpha, \lambda\right)=1$. Clearly $\sum_{i=1}^{n+1} D_{i}(\alpha, \lambda)=1$.

The maximum product of spacings estimators $\widehat{\alpha}_{M P S}$ and $\widehat{\lambda}_{M P S}$, of the parameters $\alpha$ and $\lambda$ are obtained by maximizing the geometric mean of the spacings with respect to $\alpha$ and $\lambda$

$$
G(\alpha, \lambda)=\left[\prod_{i=1}^{n+1} D_{i}(\alpha, \lambda)\right]^{\frac{1}{n+1}}
$$

or, equivalently, by maximizing the function

$$
H(\alpha, \lambda)=\frac{1}{n+1} \sum_{i=1}^{n+1} \log D_{i}(\alpha, \lambda) .
$$

The estimators $\widehat{\alpha}_{M P S}$ and $\widehat{\lambda}_{M P S}$ of the parameters $\alpha$ and $\lambda$ can be obtained by solving the nonlinear equations

$$
\begin{aligned}
& \frac{\partial}{\partial \alpha} H(\alpha, \lambda)=\frac{1}{n+1} \sum_{i=1}^{n+1} \frac{1}{D_{i}(\alpha, \lambda)}\left[\eta_{1}\left(x_{i: n} \mid \alpha, \lambda\right)-\eta_{1}\left(x_{i-1: n} \mid \alpha, \lambda\right)\right]=0 \\
& \frac{\partial}{\partial \lambda} H(\alpha, \lambda)=\frac{1}{n+1} \sum_{i=1}^{n+1} \frac{1}{D_{i}(\alpha, \lambda)}\left[\eta_{2}\left(x_{i: n} \mid \alpha, \lambda\right)-\eta_{2}\left(x_{i-1: n} \mid \alpha, \lambda\right)\right]=0
\end{aligned}
$$

where $\eta_{1}(\cdot \mid \alpha, \lambda)$ and $\eta_{2}(\cdot \mid \alpha, \lambda)$ are given by (9) and (10), respectively. 
Similarly, the minimum spacing distance estimators of $\widehat{\alpha}_{M S A D E}$ and $\widehat{\lambda}_{M S A D E}$ of $\alpha$ and $\lambda$ are obtained by minimizing

$$
T(\alpha, \lambda)=\sum_{i=1}^{n+1} h\left(D_{i}(\alpha, \lambda), \frac{1}{n+1}\right),
$$

where $h(x, y)$ is an appropriate distance. Some choices of $h(x, y)$ are the absolute distance $|x-y|$ and the absolute-log distance $|\log x-\log y|$. These estimators are called the "minimum spacing absolute distance estimator" (MSADE) and the "minimum spacing absolute-log distance estimator" (MSALDE). The MSADE and MSALDE of parameters $\alpha$ and $\lambda$ can be obtained by minimizing

$$
T(\alpha, \lambda)=\sum_{i=1}^{n+1} \mid\left(D_{i}(\alpha, \lambda)-\frac{1}{n+1} \mid\right.
$$

and

$$
T(\alpha, \lambda)=\sum_{i=1}^{n+1}\left|\log D_{i}(\alpha, \lambda)-\log \frac{1}{n+1}\right|
$$

with respect to $\alpha$ and $\lambda$, respectively.

The estimators $\hat{\alpha}_{M S A D E}$ and $\hat{\lambda}_{M S A D E}$ of $\alpha$ and $\lambda$ can be obtained by solving the following nonlinear equations

$$
\begin{aligned}
\frac{\partial}{\partial \alpha} T(\alpha, \lambda) & =\sum_{i=1}^{n+1} \frac{D_{i}(\alpha, \lambda)-\frac{1}{n+1}}{\left|D_{i}(\alpha, \lambda)-\frac{1}{n+1}\right|}\left[\eta_{1}\left(x_{i: n} \mid \alpha, \lambda\right)-\eta_{1}\left(x_{i-1: n} \mid \alpha, \lambda\right)\right]=0 \\
\frac{\partial}{\partial \lambda} T(\alpha, \lambda) & =\sum_{i=1}^{n+1} \frac{D_{i}(\alpha, \lambda)-\frac{1}{n+1}}{\left|D_{i}(\alpha, \lambda)-\frac{1}{n+1}\right|}\left[\eta_{2}\left(x_{i: n} \mid \alpha, \lambda\right)-\eta_{2}\left(x_{i-1: n} \mid \alpha, \lambda\right)\right]=0,
\end{aligned}
$$

where $D_{i}(\alpha, \lambda) \neq \frac{1}{n+1}$.

The estimators $\hat{\alpha}_{M S A L D E}$, and $\hat{\lambda}_{M S A L D E}$ of $\alpha$ and $\lambda$ can be obtained by solving the nonlinear equations

$$
\begin{aligned}
\frac{\partial}{\partial \alpha} T(\alpha, \lambda)=\sum_{i=1}^{n+1} \frac{\log D_{i}(\alpha, \lambda)-\log \frac{1}{n+1}}{\left|\log D_{i}(\alpha, \lambda)-\log \frac{1}{n+1}\right|} \frac{1}{D_{i}(\alpha, \lambda)} \\
\quad \times\left[\eta_{1}\left(x_{i: n} \mid \alpha, \lambda\right)-\eta_{1}\left(x_{i-1: n} \mid \alpha, \lambda\right)\right]=0 \\
\frac{\partial}{\partial \lambda} T(\alpha, \lambda)=\sum_{i=1}^{n+1} \frac{\log D_{i}(\alpha, \lambda)-\log \frac{1}{n+1}}{\mid \begin{array}{l}
\log D_{i}(\alpha, \lambda)-\log \frac{1}{n+1} \mid \\
D_{i}(\alpha, \lambda)
\end{array}} \\
\quad \times\left[\eta_{2}\left(x_{i: n} \mid \alpha, \lambda\right)-\eta_{2}\left(x_{i-1: n} \mid \alpha, \lambda\right)\right]=0,
\end{aligned}
$$

where $\log D_{i}(\alpha, \lambda) \neq \log \frac{1}{n+1}$. 
3.5. Minimum Distances Estimators. This section presents three estimation methods for $\alpha$ and $\lambda$ based on the minimization of the goodness-of-fit statistics with respect to $\alpha$ and $\lambda$. This class of statistics is based on the difference between the estimate of the cumulative distribution function and the empirical distribution function.

3.5.1. Cramér-von-Mises Estimators. To motivate our choice of Cramér-von-Mises type minimum distance estimators, 31. provided empirical evidence that the bias of the estimator is smaller than the other minimum distance estimators. Thus, the Cramér-von Mises estimators $\widehat{\alpha}_{C M E}$ and $\widehat{\lambda}_{C M E}$ of the parameters $\alpha$ and $\lambda$ are obtained by minimizing the following function.

$$
C(\alpha, \lambda)=\frac{1}{12 n}+\sum_{i=1}^{n}\left(F\left(x_{i: n} \mid \alpha, \lambda\right)-\frac{2 i-1}{2 n}\right)^{2}
$$

These estimators can be obtained by solving the following non-linear equations

$$
\begin{aligned}
& \sum_{i=1}^{n}\left(F\left(x_{i: n} \mid \alpha, \lambda\right)-\frac{2 i-1}{2 n}\right) \eta_{1}\left(x_{i: n} \mid \alpha, \lambda\right)=0 \\
& \sum_{i=1}^{n}\left(F\left(x_{i: n} \mid \alpha, \lambda\right)-\frac{2 i-1}{2 n}\right) \eta_{2}\left(x_{i: n} \mid \alpha, \lambda\right)=0
\end{aligned}
$$

where $\eta_{1}(\cdot \mid \alpha, \lambda)$ and $\eta_{2}(\cdot \mid \alpha, \lambda)$ are given by (9) and 10 respectively.

3.5.2. Anderson-Darling and Right-tail Anderson-Darling Estimators. The AndersonDarling (AD) test [32] is an alternative method to detect sample distribution departure from the assumed distribution. Specifically, the AD test converge very quickly towards the asymptote [33, 34, 35]. The Anderson-Darling estimators $\widehat{\alpha}_{A D E}$ and $\widehat{\lambda}_{A D E}$ of the parameters $\alpha$ and $\lambda$ are obtained by minimizing the following function with respect to the parameters.

$$
A(\alpha, \lambda)=-n-\frac{1}{n} \sum_{i=1}^{n}(2 i-1)\left\{\log F\left(x_{i: n} \mid \alpha, \lambda\right)+\log \bar{F}\left(x_{n+1-i: n} \mid \alpha, \lambda\right)\right\}
$$

These estimators can be obtained by solving the following non-linear equations:

$$
\begin{aligned}
& \sum_{i=1}^{n}(2 i-1)\left[\frac{\eta_{1}\left(x_{i: n} \mid \alpha, \lambda\right)}{F\left(x_{i: n} \mid \alpha, \lambda\right)}-\frac{\eta_{1}\left(x_{n+1-i: n} \mid \alpha, \lambda\right)}{\bar{F}\left(x_{n+1-i: n} \mid \alpha, \lambda\right)}\right]=0 \\
& \sum_{i=1}^{n}(2 i-1)\left[\frac{\eta_{2}\left(x_{i: n} \mid \alpha, \lambda\right)}{F\left(x_{i: n} \mid \alpha, \lambda\right)}-\frac{\eta_{2}\left(x_{n+1-i: n} \mid \alpha, \lambda\right)}{\bar{F}\left(x_{n+1-i: n} \mid \alpha, \lambda\right)}\right]=0
\end{aligned}
$$

where $\eta_{1}(\cdot \mid \alpha, \lambda)$ and $\eta_{2}(\cdot \mid \alpha, \lambda)$ are given by $(9)$ and 10, , respectively. 
The Right-tail Anderson-Darling estimators $\widehat{\alpha}_{R T A D E}$ and $\widehat{\lambda}_{R T A D E}$ of the parameters $\alpha$ and $\lambda$ are obtained by minimizing, with respect to $\alpha$ and $\lambda$, the function:

$$
R(\alpha, \lambda)=\frac{n}{2}-2 \sum_{i=1}^{n} F\left(x_{i: n} \mid \alpha, \lambda\right)-\frac{1}{n} \sum_{i=1}^{n}(2 i-1) \log \bar{F}\left(x_{n+1-i: n} \mid \alpha, \lambda\right) .
$$

Equivalently

$$
\begin{aligned}
& -2 \sum_{i=1}^{n} \eta_{1}\left(x_{i: n} \mid \alpha, \lambda\right)+\frac{1}{n} \sum_{i=1}^{n}(2 i-1) \frac{\eta_{1}\left(x_{n+1-i: n} \mid \alpha, \lambda\right)}{\bar{F}\left(x_{n+1-i: n} \mid \alpha, \lambda\right)}=0 \\
& -2 \sum_{i=1}^{n} \eta_{2}\left(x_{i: n} \mid \alpha, \lambda\right)+\frac{1}{n} \sum_{i=1}^{n}(2 i-1) \frac{\eta_{2}\left(x_{n+1-i: n} \mid \alpha, \lambda\right)}{\bar{F}\left(x_{n+1-i: n} \mid \alpha, \lambda\right)}=0
\end{aligned}
$$

where $\eta_{1}(\cdot \mid \alpha, \lambda)$ and $\eta_{2}(\cdot \mid \alpha, \lambda)$ are given by $(9)$ and $(10)$, respectively.

\section{BAYESIAN ANALYSIS}

This section discusses the Bayesian estimation of the FW distribution. To this end, the likelihood function can be written as

$L(\alpha, \lambda \mid \boldsymbol{x})=\exp \left(\sum_{i=1}^{n} \log \left(\alpha+\lambda / x_{i}^{2}\right)\right) \exp \left(\alpha \sum_{i=1}^{n} x_{i}-\lambda \sum_{i=1}^{n} x_{i}^{-1}\right) \exp \left(-\sum_{i=1}^{n} \exp \left(\alpha x_{i}-\lambda / x_{i}\right)\right)$

Next assuming $\alpha \sim \operatorname{Gamma}(a, b)$, i.e., $f(\alpha)=\frac{b^{a}}{\Gamma(a)} \alpha^{a-1} \exp (-b \alpha)$, and $\lambda \sim$ $\operatorname{Gamma}(c, d)$, the joint posterior of $\alpha$ and $\lambda$ can be written as

$$
\begin{aligned}
P(\alpha, \lambda \mid \boldsymbol{x}) \propto \quad & \alpha^{a-1} \exp \left(-\alpha\left(b-\sum_{i=1}^{n} x_{i}\right)\right) \lambda^{c-1} \exp \left(-\lambda\left(d+\sum_{i=1}^{n} x_{i}^{-1}\right)\right) \\
& \times \exp \left(\sum_{i=1}^{n} \log \left(\alpha+\lambda / x_{i}^{2}\right)-\sum_{i=1}^{n} \exp \left(\alpha x_{i}-\lambda / x_{i}\right)\right)
\end{aligned}
$$

The marginal distribution of $\lambda$ is $P(\lambda \mid \boldsymbol{x}) \sim \operatorname{Gamma}\left(c, d+\sum_{i=1}^{n} x_{i}^{-1}\right)$ while $P(\alpha \mid \lambda, \boldsymbol{x}) \sim$ $\alpha^{a-1} \exp \left(-\alpha\left(b-\sum_{i=1}^{n} x_{i}\right)\right) \exp \left(\sum_{i=1}^{n} \log \left(\alpha+\lambda / x_{i}^{2}\right)-\sum_{i=1}^{n} \exp \left(\alpha x_{i}-\lambda / x_{i}\right)\right)$ for

To generate marginal of $\alpha$, we propose the adaptive rejection sampling. To this end, it is not difficult to show that $P(\alpha \mid \lambda, \boldsymbol{x})$ is log-concave and thus, the idea of [36] can be used. For Metropolis Hastings (MH) sampling, we assume the gamma density as transition kernel $q\left(\alpha^{(i)} \mid \alpha^{(*)}\right)$ for sampling value of $\alpha$. The choice of gamma distribution has been done purely for illustration purpose, and other suitable distributions can be considered. After generating the marginal densities, the next step is to calculate the posterior summaries, $\mathbb{E}(\boldsymbol{\theta} \mid \boldsymbol{x})=\int_{\boldsymbol{\theta}} \boldsymbol{\theta} \mathbb{P}(\boldsymbol{\theta} \mid \boldsymbol{x})$. The steps to calculate the Bayes estimates are as follow:

MH Algorithm-Step 1: Generate $\lambda$ from the Gamma distribution. 
(1) To generate the $\alpha$, evaluate the acceptance probability by $k\left(\alpha^{(i)}, \alpha^{(*)}\right)=$ $\min \left(1, \frac{P\left(\alpha^{(*)} \mid \boldsymbol{x}\right) q\left(\alpha^{(i)} \mid \alpha^{(*)}\right)}{P\left(\alpha^{(i)} \mid \boldsymbol{x}\right) q\left(\alpha^{(*)} \mid \alpha^{(i)}\right)}\right)$, where $P(\alpha \mid \boldsymbol{x}, \lambda)$ has been defined above.

(2) Generate a random $u$ from $U$ niform $(0,1)$

(3) If $k\left(\alpha^{(i)}, \alpha^{(*)}\right) \geq u, \alpha^{(i+1)}=\alpha^{(*)}$, otherwise $\alpha^{(i+1)}=\alpha^{(i)}$.

Step 2: Suppose at the i-th step, $\alpha$ and $\lambda$ take the values $\alpha_{i}$ and $\lambda_{i}$ and we can generate $\mathbb{P}\left(\lambda_{i+1} \mid \boldsymbol{x}\right)$, and $\mathbb{P}\left(\alpha_{i+1} \mid \lambda_{i}, \boldsymbol{x}\right)$;

Step 3: Repeat the above step $N$ times;

Step 4: Calculate the Bayes estimator of $g(\alpha, \lambda)$ by $\frac{1}{N-M} \sum_{i=M+1}^{N} g\left(\alpha_{i}, \lambda_{i}\right)$, where $M$ denotes the burn-in sample.

In the next section, a simulation study is done to assess the performance of different estimation methods.

\section{Simulation Study}

This section presents Monte Carlo simulation studies to assess the performance of the frequentist estimators derived in the previous section. In particular, we use bias, the root mean squared error, the average absolute difference between the theoretical and the empirical estimate of the distribution functions, and the maximum absolute difference between the theoretical and empirical distribution functions as the performance assessment criteria. For comparison, we considered the following sample sizes: $n=20,40,60,80,100$. Ten thousand independent samples of the aforementioned sizes were generated from EW distribution with parameters $(\alpha, \lambda)=\{(0.5,0.5),(1.5,0.5),(1.5,2.0),(3.0,2.0)\}$. It is noticed that 10,000 repetitions are sufficiently large to have stable results. For all the methods considered in this study, first we estimated the parameters using the method of maximum likelihood and then these estimates are used as the initial values. Since the MLE are not in closed form, we used the 'fitdist' function of $\mathrm{R}$ package fitdistrplus, which optimized the logarithm of the likelihood function numerically, to estimate the parameters. The results of the simulation studies are tabulated in Tables 144.

For each estimate, we calculated the bias, the root mean-squared error (RMSE), the average absolute difference between the theoretical and the empirical estimate of the distribution functions $\left(D_{a b s}\right)$, and the maximum absolute difference between the theoretical and the empirical distribution functions $\left(D_{\max }\right)$. The statistics are obtained using the following formulae:

$$
\begin{gathered}
\operatorname{Bias}(\hat{\alpha})=\frac{1}{K} \sum_{i=1}^{K}\left(\hat{\alpha}_{i}-\alpha\right), \quad \operatorname{Bias}(\hat{\lambda})=\frac{1}{K} \sum_{i=1}^{K}\left(\hat{\lambda}_{i}-\lambda\right) \\
\operatorname{RMSE}(\hat{\alpha})=\sqrt{\frac{1}{K} \sum_{i=1}^{K}\left(\hat{\alpha}_{i}-\alpha\right)^{2},} \quad \operatorname{RMSE}(\hat{\lambda})=\sqrt{\frac{1}{K} \sum_{i=1}^{K}\left(\hat{\lambda}_{i}-\lambda\right)^{2}}
\end{gathered}
$$




$$
\begin{aligned}
& D_{\text {abs }}(\hat{\alpha})=\frac{1}{(n K)} \sum_{i=1}^{K} \sum_{j=1}^{n}\left|F\left(x_{i j} \mid \alpha, \lambda\right)-F\left(x_{i j} \mid \hat{\alpha}, \hat{\lambda}\right)\right| \\
& D_{\max }(\hat{\alpha})=\frac{1}{n K} \sum_{i=1}^{K} \max _{j}\left|F\left(x_{i j} \mid \alpha, \lambda\right)-F\left(x_{i j} \mid \hat{\alpha}, \hat{\lambda}\right)\right|
\end{aligned}
$$

where $\mathrm{n}$ denotes the sample size and $\mathrm{K}$ is the number of iterations. Simulated bias, RMSE, $D_{\text {abs }}, D_{\max }$ for the estimates are given in Tables 1.4. The row with label $\sum$ Ranks shows the partial sum of the ranks and superscript indicates the rank of each of the estimators among all the estimators for that metric. For example, Table-1 shows the bias of $\operatorname{MLE}(\hat{\alpha})$ as $1.731^{8}$ for $n=20$. This indicates, bias of $\hat{\alpha}$ obtained using the method of maximum likelihood ranks $8^{\text {th }}$ among all other estimators.

The following observations can be drawn from the Tables 1.4.

1. All the estimators show the property of consistency, i.e., the RMSE decreases as the sample size increases, except in the case of PCE and MSALDE for $\alpha=0.5$. However, assuming $\alpha>1$, the RMSE of MSALDE decreases by increasing the sample size. Furthermore, assuming $\alpha=1.5, \lambda=0.5$, the RMSE of assuming $\alpha$ increases with the sample size for the MLE.

2. The bias of $\hat{\alpha}$ and $\hat{\lambda}$ decreases with increasing $n$ for all the method of estimations. 3 . It is noticed that the MLE and PCE performed the worst than the rest methods. The MSALDE performs the best when $\alpha, \lambda>1$. The CVM and AD are suggested only when $\alpha>1$.

4. $D_{\text {abs }}$ is smaller than $D_{\max }$ for all the estimation techniques. Again, the statistics gets smaller with the increase of sample size.

5 . In terms of performance of the methods of estimation, the MSADE and AD estimators uniformly produces the least biases of the estimates with the least RMSE, see the ranking of $\sum$ Ranks rows in the tables, for the most configurations considered in our studies.

6. It is also observed that for the estimation of $\lambda$, PCE performed the worst, as the RMSE is the highest as compared to the other methods.

For the Bayesian analysis, we generated 12,000 samples of $\alpha$ and $\lambda$, and the Bayes estimates with other posterior summaries, like MCMC error, median, 95\% Bayesian intervals have been tabulated in Table-5. For the parameter combinations mentioned above to compute the posterior summaries, hyperparameters are selected in such a way that the mean of the priors equal to the parameters' nominal values with large variances. Moreover, we used $M=2,000$ as a burn-in period for our calculations. From the table, it is clear that as the sample size increases, the Bayes estimates approaches to the nominal values and the Bayesian intervals become more smaller for large sample sizes. Furthermore, the MCMC error decreases with the increase of sample size. 
TABLE 1. Simulation results for $\alpha=\lambda=0.5$.

\begin{tabular}{|c|c|c|c|c|c|c|c|c|c|c|c|}
\hline$n$ & & & LSE & WLS & $\mathrm{PCE}$ & & & & & $\mathrm{AD}$ & \\
\hline \multirow[t]{7}{*}{20} & & $731^{8}$ & $1.680^{7}$ & $12.813^{10}$ & $-0.381^{2}$ & $1.409^{5}$ & $-0.385^{3}$ & $-0.311^{1}$ & $1.893^{9}$ & $1.101^{4}$ & $1.629^{6}$ \\
\hline & $\operatorname{RMSE}(\hat{\alpha})$ & $820^{7}$ & $1.925^{8}$ & $14.131^{10}$ & $0.381^{2}$ & $1.492^{5}$ & $0.389^{3}$ & $0.373^{1}$ & $2.159^{9}$ & $1.184^{4}$ & $1.724^{6}$ \\
\hline & $\operatorname{Bias}(\hat{\lambda})$ & $365^{4}$ & $-0.367^{5}$ & $0.000^{1}$ & $29.943^{10}$ & $-0.377^{7}$ & $425^{8}$ & $12.480^{9}$ & $-0.360^{2}$ & $-0.374^{6}$ & $-0.363^{3}$ \\
\hline & $\operatorname{RMSE}(\hat{\lambda})$ & $367^{4}$ & $0.369^{5}$ & & $2.900^{10}$ & $0.378^{6}$ & & & $0.362^{2}$ & & $0.367^{3}$ \\
\hline & $D_{\text {abs }}$ & $0.363^{10}$ & $0.359^{6}$ & $0.327^{5}$ & $0.137^{3}$ & $0.360^{7}$ & & & $0.361^{8}$ & $0.315^{4}$ & $0.361^{9}$ \\
\hline & & $0.528^{8}$ & $517^{6}$ & $0.777^{10}$ & $0.495^{4}$ & $0.500^{5}$ & & & $0.533^{9}$ & $0.442^{1}$ & $0.517^{7}$ \\
\hline & $\sum \mathrm{Ra}$ & $41^{10}$ & & & $31^{4}$ & $35^{6}$ & & & $39^{9}$ & $26^{3}$ & \\
\hline \multirow[t]{7}{*}{40} & Bias & $1.608^{8}$ & $1.565^{7}$ & $11.733^{10}$ & $-0.498^{3}$ & $1.419^{5}$ & & & $1.665^{9}$ & $1.064^{4}$ & $1.559^{6}$ \\
\hline & RMS & $64:$ & $1.649^{8}$ & $12.976^{10}$ & $0.498^{2}$ & & & & & $102^{4}$ & \\
\hline & Bias & .378 & $-0.372^{5}$ & $0.000^{1}$ & $36.321^{10}$ & $.378^{6}$ & & & & & \\
\hline & RMSE & 0.37 & 0.37 & & & & & & & & \\
\hline & & 10 & $0.361^{6}$ & 0.32 & $0.137^{3}$ & & & & & & $0.362^{8}$ \\
\hline & & & $0.513^{3}$ & 0.78 & & & & & & & 0.5 \\
\hline & & & & & & & & & & & \\
\hline \multirow[t]{7}{*}{60} & & & $1.533^{6}$ & $11.14 !$ & $-0.399^{2}$ & & & & & $1.051^{4}$ & $1.537^{7}$ \\
\hline & & & $582^{7}$ & 12.27 & $0.39 !$ & & & & & & \\
\hline & & 4 & $-0.373^{5}$ & $0.000^{1}$ & 10 & & & & & & $-0.372^{4}$ \\
\hline & RM & & & & & & & & & & \\
\hline & & & & & 0.1 & & & & & & $32^{7}$ \\
\hline & & & 51 & 0.77 & 0.56 & & & & & 0.43 & 0.5 \\
\hline & & & & & & & & & & & \\
\hline \multirow[t]{7}{*}{80} & & & $520^{6}$ & & $-0.403^{3}$ & & & & & & $27^{7}$ \\
\hline & & & $554^{7}$ & 11.7 & $0.403^{2}$ & & & & & & $6^{6}$ \\
\hline & & -0 & $-0.374^{5}$ & 0.0 & 58.68 & & & & -0.3 & -0.3 & \\
\hline & & & & & & & & & & & \\
\hline & & & & & & & & & & & \\
\hline & & & & & 0.5 & & & & & & \\
\hline & & & & & & & & & & & \\
\hline \multirow[t]{7}{*}{100} & & & & & & & & & & & \\
\hline & & & $1.541^{7}$ & 11.40 & $0.405^{2}$ & & & & & $56^{4}$ & \\
\hline & & & $-0.374^{5}$ & & $57.713^{10}$ & & & & -0.3 & -0.3 & \\
\hline & & & & & & & & & & & \\
\hline & & & & & & & & & & & \\
\hline & & & $0.510^{3}$ & & & 0.50 & & & & & \\
\hline & & & & & & & & & & & \\
\hline
\end{tabular}

\section{Data Analysis}

This section shows empirically that the FW distribution can be used as an alternative to some well-known two-parameter models like gamma, log-normal, Weibull, exponentiated exponential (EE), Nadarajah and Haghighi (NH) 37, BirnbaumSaunders (BS), and inverse Gaussian (IG) distributions. For model comparison, we consider three well-known statistics and three model selection criteria. These measures and selection criteria are: Anderson-Darling $\left(A^{*}\right)$, Cramér-von Mises $\left(W^{*}\right)$ and Kolmogorov-Smirnov (K-S) measures, Akaike information criterion (AIC),Bayesian information criterion (BIC), and loglikelihood. The least value of these measures and selection criteria may indicate better fit. The cdfs of the EE, NH, BS and pdf 
TABLE 2. Simulation results for $\alpha=1.5, \lambda=0.5$.

\begin{tabular}{|c|c|c|c|c|c|c|c|c|c|c|c|}
\hline & Est. & MLE & LSE & WLS & PCE & MPS & MSADF & MSALDF & CVM & AD & RAD \\
\hline \multirow{7}{*}{$\frac{n}{20}$} & $\operatorname{Bias}(\hat{\alpha})$ & $-0.770^{4}$ & $-0.824^{6}$ & $-0.817^{5}$ & $-1.487^{8}$ & $-0.857^{7}$ & & & & $-0.335^{2}$ & $-0.321^{1}$ \\
\hline & $\operatorname{RMSE}(\hat{\alpha})$ & $0.785^{3}$ & $0.847^{6}$ & $0.837^{5}$ & $1.487^{8}$ & $0.897^{7}$ & $3.236^{9}$ & $3.468^{10}$ & $0.789^{4}$ & $0.390^{2}$ & $0.380^{1}$ \\
\hline & $\operatorname{Bias}(\hat{\lambda})$ & $0.715^{8}$ & $0.642^{6}$ & $0.650^{7}$ & $121.751^{10}$ & $0.565^{5}$ & $-0.225^{2}$ & $0.038^{1}$ & $0.723^{9}$ & $0.245^{3}$ & $0.271^{4}$ \\
\hline & $\operatorname{RMSE}(\hat{\lambda})$ & $0.773^{6}$ & $0.717^{4}$ & $0.719^{5}$ & $175.811^{10}$ & $0.622^{3}$ & $1.735^{9}$ & $1.435^{8}$ & $0.803^{7}$ & $0.312^{1}$ & $0.359^{2}$ \\
\hline & $D_{\text {abs }}$ & $0.332^{7}$ & $0.332^{9}$ & $0.332^{8}$ & $0.831^{10}$ & $0.325^{5}$ & $0.162^{4}$ & $0.110^{1}$ & $0.331^{6}$ & $0.154^{2}$ & $0.156^{3}$ \\
\hline & & $0.497^{8}$ & $0.486^{5}$ & $0.487^{6}$ & & $0.470^{4}$ & $0.581^{9}$ & $0.356^{3}$ & & $0.218^{1}$ & $0.225^{2}$ \\
\hline & $\sum \mathrm{Ral}$ & $36^{6.5}$ & $36^{6.5}$ & & & & & & & & \\
\hline \multirow[t]{7}{*}{40} & $\operatorname{Bias}(\hat{\alpha})$ & $-0.803^{4}$ & $-0.831^{7}$ & $-0.825^{6}$ & $-1.488^{8}$ & $-0.819^{5}$ & $3.290^{10}$ & $1.953^{9}$ & $-0.800^{3}$ & $-0.174^{2}$ & $-0.168^{1}$ \\
\hline & RMSE & $0.809^{3}$ & $0.840^{6}$ & $0.833^{5}$ & $1.488^{8}$ & $1.102^{7}$ & $3.891^{10}$ & & & $0.228^{2}$ & $0.221^{1}$ \\
\hline & Bias & $0.667^{7}$ & $0.631^{5}$ & $0.638^{6}$ & $143.170^{10}$ & $0.578^{4}$ & $-0.340^{3}$ & & 0.66 & $0.115^{1}$ & $0.125^{2}$ \\
\hline & $\operatorname{RMSE}(\hat{\lambda})$ & $0.695^{6}$ & $0.666^{4}$ & $0.670^{5}$ & 207.4 & & & & & $0.159^{1}$ & \\
\hline & $D_{\mathrm{ab}}$ & $332^{7}$ & $0.332^{8}$ & $0.332^{9}$ & 0.83 & & & & & $0.082^{1}$ & \\
\hline & & $0.494^{8}$ & $0.488^{5}$ & $0.489^{6}$ & & & & & & & $0.121^{2}$ \\
\hline & $\sum \mathrm{R}$ & & & & & & & & & & \\
\hline \multirow[t]{7}{*}{60} & & $-0.814^{6}$ & $-0.832^{8}$ & $-0.827^{7}$ & $-1.488^{9}$ & $-0.740^{4}$ & $3.846^{10}$ & & $-0.812^{5}$ & $-0.086^{2}$ & $082^{1}$ \\
\hline & RMS & $0.817^{3}$ & $0.838^{6}$ & $0.832^{5}$ & & & & & & $0.151^{2}$ & \\
\hline & & 0.65 & 0.62 & $0.635^{6}$ & 159. & 0.5 & & & & $0.057^{1}$ & $63^{2}$ \\
\hline & $\operatorname{RMSE}(\hat{\lambda})$ & $0.671^{6}$ & $0.651^{4}$ & $0.655^{5}$ & 227 & 0.6 & 1.2 & & 0.6 & $0.099^{1}$ & \\
\hline & & $0.332^{8}$ & $0.332^{6}$ & $0.332^{7}$ & 0.8 & & & & & $0.047^{1}$ & $0.049^{2}$ \\
\hline & & $0.4 \mathrm{~s}-\mathrm{s}$ & $0.488^{4}$ & $0.490^{5}$ & & 0.47 & & & & $0.070^{1}$ & \\
\hline & & & & & & & & & & & \\
\hline \multirow[t]{7}{*}{80} & Bia & $-0.819^{6}$ & $-0.833^{8}$ & $-0.829^{7}$ & & $-0.663^{4}$ & & & & $-0.089^{2}$ & $-0.086^{1}$ \\
\hline & $\operatorname{RMSE}(\hat{\alpha})$ & & 0.83 & & & & & & & $0.139^{2}$ & \\
\hline & & 0.6 & 0.62 & $0.632^{6}$ & 177 . & & & & & 0.0 & 0.06 \\
\hline & $\operatorname{RMSE}(\hat{\lambda})$ & $0.658^{6}$ & 0.64 & $0.648^{5}$ & 259. & & & & & $0.089^{1}$ & 0.10 \\
\hline & & $0.332^{7}$ & $0.332^{6}$ & $0.332^{8}$ & $0.832^{10}$ & $0.325^{4}$ & $0.168^{3}$ & & $0.332^{5}$ & $0.044^{1}$ & $0.046^{2}$ \\
\hline & & $0.492^{7}$ & & & & & & & & & \\
\hline & & & & & & & & & & & \\
\hline \multirow{7}{*}{00} & Bias & $-0.822^{6}$ & $-0.833^{8}$ & $-0.829^{7}$ & $-1.489^{9}$ & -0. & & $0.314^{3}$ & $-0.821^{5}$ & $-0.091^{2}$ & -0.089 \\
\hline & RMS & & 0.83 & & & & & & & & \\
\hline & & & 0.62 & $0.631^{6}$ & 190. & & & & & 0.0 & \\
\hline & $\operatorname{RMSE}(\hat{\lambda})$ & 0 & 0.64 & $0.643^{5}$ & 271. & & & & 0.6 & $0.083^{1}$ & $0.093^{2}$ \\
\hline & & & $0.332^{6}$ & & & & & & & $0.043^{1}$ & $0.044^{2}$ \\
\hline & & & $0.489^{4}$ & & & 0.47 & & & 0.4 & 0.06 & \\
\hline & & & & & & & & & & & \\
\hline
\end{tabular}

of the IG distributions are, respectively, given by

$$
\begin{aligned}
F_{E E}(x ; \alpha, \lambda) & =\left(1-\mathrm{e}^{-\lambda \mathrm{x}}\right)^{\alpha}, \quad x, \theta>0, \\
F_{N H}(x ; \alpha, \lambda) & =1-\mathrm{e}^{1-(1+\lambda x)^{\alpha}}, \quad x, \alpha, \lambda>0, \\
F_{B S}(x ; \alpha, \beta) & =\Phi\left[\frac{1}{\alpha}\left\{\left(\frac{x}{\beta}\right)^{1 / 2}-\left(\frac{\beta}{x}\right)^{1 / 2}\right\}\right], \quad x, \alpha,>0, \\
f_{I G}(x ; \mu, \lambda) & =\sqrt{\frac{\lambda}{2 \pi x^{3}}} \exp \left[-\lambda(x-\mu)^{2} /\left(2 x \mu^{2}\right)\right], \quad x, \mu, \lambda>0 .
\end{aligned}
$$

6.1. Strength of glass fibres. This data set corresponds to the strengths of $15 \mathrm{~cm}$ fibres and taken from [38. The data are: $0.37,0.40,0.70,0.75,0.80,0.81,0.83,0.86$, $0.92,0.92,0.94,0.95,0.98,1.03,1.06,1.06,1.08,1.09,1.10,1.10,1.13,1.14,1.15$, $1.17,1.20,1.20,1.21,1.22,1.25,1.28,1.28,1.29,1.29,1.30,1.35,1.35,1.37,1.37$, $1.38,1.40,1.40,1.42,1.43,1.51,1.53,1.61$. A summary of these data is: $\mathrm{n}=46, \bar{x}$ 
TABLE 3. Simulation results for $\alpha=1.5, \lambda=2.0$.

\begin{tabular}{|c|c|c|c|c|c|c|c|c|c|c|c|}
\hline & & MLE & LSE & WLS & & MPS & MSADF & 1SALD & $v_{111}$ & AD & RAD \\
\hline \multirow[t]{7}{*}{$\frac{n}{20}$} & $\operatorname{Bias}(\hat{\alpha})$ & $-0.779^{7}$ & $-0.833^{9}$ & $-0.826^{8}$ & $-1.467^{10}$ & $-0.523^{5}$ & $0.253^{3}$ & & $-0.774^{6}$ & $0.242^{2}$ & $0.264^{4}$ \\
\hline & $\operatorname{RMSE}(\hat{\alpha})$ & $0.791^{5}$ & $0.849^{8}$ & $0.840^{7}$ & $1.468^{9}$ & $2.258^{10}$ & $0.775^{4}$ & $0.408^{3}$ & $0.795^{6}$ & $0.335^{1}$ & $0.364^{2}$ \\
\hline & & $2.839^{8}$ & $2.514^{6}$ & $2.554^{7}$ & $297.882^{10}$ & $2.245^{5}$ & $1.455^{4}$ & $-0.102^{1}$ & $2.855^{9}$ & $-0.337^{3}$ & $-0.291^{2}$ \\
\hline & $\operatorname{RMSE}(\hat{\lambda})$ & $3.024^{7}$ & $2.764^{5}$ & $2.779^{6}$ & $416.378^{10}$ & $2.515^{4}$ & $5.565^{9}$ & $0.382^{1}$ & $3.123^{8}$ & $0.512^{2}$ & $0.536^{3}$ \\
\hline & $D_{\text {abs }}$ & $0.456^{9}$ & $0.456^{8}$ & $0.455^{7}$ & $0.955^{10}$ & $0.437^{5}$ & $0.110^{2}$ & $0.012^{1}$ & $0.455^{6}$ & $0.146^{3}$ & $0.146^{4}$ \\
\hline & & $0.796^{9}$ & $0.778^{6}$ & $0.781^{7}$ & $1.000^{10}$ & $0.751^{5}$ & $0.260^{4}$ & $0.088^{1}$ & $0.793^{8}$ & $0.218^{2}$ & $0.219^{3}$ \\
\hline & $\sum \mathrm{R}$ & $45^{9}$ & & & & & & & & & \\
\hline \multirow[t]{7}{*}{40} & $\operatorname{Bias}(\hat{\alpha})$ & $-0.807^{7}$ & $-0.835^{9}$ & $-0.829^{8}$ & $-1.470^{10}$ & $0.323^{5}$ & $0.271^{4}$ & $0.074^{1}$ & $-0.807^{6}$ & $0.226^{2}$ & $0.235^{3}$ \\
\hline & RMSE & $0.812^{4}$ & $0.842^{8}$ & $0.835^{7}$ & $1.470^{9}$ & $4.616^{10}$ & $0.829^{6}$ & & $0.814^{5}$ & $0.274^{2}$ & \\
\hline & Bias & $2.661^{9}$ & $2.497^{6}$ & $2.532^{7}$ & $359.246^{10}$ & $2.394^{5}$ & $1.856^{4}$ & $-0.106^{1}$ & $2.659^{8}$ & $-0.360^{3}$ & $-0.340^{2}$ \\
\hline & $\operatorname{RMSE}(\hat{\lambda})$ & $2.748^{7}$ & $2.612^{5}$ & $2.633^{6}$ & $489.784^{10}$ & $2.609^{4}$ & $6.308^{9}$ & & $2.778^{8}$ & $0.446^{2}$ & 0.4 \\
\hline & $D_{\mathrm{al}}$ & $0.455^{9}$ & $0.455^{7}$ & $0.455^{8}$ & 0.95 & $0.426^{5}$ & & & & $0.145^{4}$ & \\
\hline & & $0.798^{9}$ & & $0.791^{7}$ & & $0.765^{5}$ & & & & $0.212^{2}$ & \\
\hline & & & & & & & & & & $15^{2}$ & \\
\hline \multirow[t]{7}{*}{60} & Bias & $-0.817^{6}$ & $-0.835^{8}$ & $-0.830^{7}$ & $-1.470^{10}$ & $1.062^{9}$ & $0.464^{3}$ & & $-0.816^{5}$ & $0.460^{2}$ & $0.467^{4}$ \\
\hline & RMS & $0.819^{4}$ & $0.839^{7}$ & $0.833^{6}$ & & $5.858^{10}$ & & & & $0.482^{2}$ & \\
\hline & & $2.605^{9}$ & $2.496^{6}$ & $2.526^{7}$ & 378.6 & $2.483^{5}$ & $0.597^{2}$ & & $2.602^{8}$ & $-0.710^{4}$ & \\
\hline & RMS & $2.661^{6}$ & 2.57 & 2.5 & 514. & $2.739^{8}$ & & & $2.679^{7}$ & 0.7 & \\
\hline & & $0.456^{9}$ & 0.45 & $0.455^{8}$ & & $0.412^{5}$ & & & & 0.2 & \\
\hline & & $0.799^{9}$ & $0.792^{6}$ & $0.794^{7}$ & 1.000 & $0.767^{5}$ & $0.348^{2}$ & 0.0 & $0.798^{8}$ & $0.399^{3}$ & $0.400^{4}$ \\
\hline & & $43^{9}$ & & & & & & & & & \\
\hline \multirow[t]{7}{*}{80} & Bias & $-0.821^{6}$ & $-0.835^{8}$ & $-0.830^{7}$ & $-1.471^{9}$ & $1.735^{10}$ & $0.575^{4}$ & 0.0 & $-0.821^{5}$ & $0.457^{2}$ & \\
\hline & & $0.823^{4}$ & & 0.83 & & & & & & & \\
\hline & & $2.575^{9}$ & 2.49 & $2.519^{6}$ & 393.7 & $2.548^{7}$ & -0.04 & & $2.572^{8}$ & $-0.713^{4}$ & \\
\hline & $\operatorname{RMSE}(\hat{\lambda})$ & $2.618^{6}$ & 2.55 & 2.56 & 535. & & & & 2.6 & 0.7 & \\
\hline & & $0.455^{9}$ & 0.45 & 0 & & & & & & & \\
\hline & & $0.798^{9}$ & $0.794^{6}$ & 0.7 & 1.0 & $0.770^{5}$ & 0.36 & 0.0 & & $9_{5}^{3}$ & \\
\hline & & & & & & & & & & & \\
\hline \multirow{7}{*}{100} & $\operatorname{Bias}(\hat{\alpha})$ & $-0.824^{5}$ & $-0.835^{8}$ & $-0.831^{7}$ & $-1.471^{9}$ & $2.478^{10}$ & & & $-0.824^{6}$ & $0.456^{2}$ & $0.460^{3}$ \\
\hline & $\operatorname{RMSE}(\hat{\alpha})$ & & & & & & & & & & \\
\hline & & $2.559^{8}$ & 2.49 & $2.516^{6}$ & $410.757^{10}$ & $2.619^{9}$ & -0.3 & & $2.556^{7}$ & $-0.715^{4}$ & $-0.709^{3}$ \\
\hline & $\operatorname{RMSE}(\hat{\lambda})$ & $593^{7}$ & 2.538 & $2.555^{6}$ & $546.167^{10}$ & & & & & 0.7 & \\
\hline & & $0.455^{9}$ & 0.45 & $0.455^{8}$ & & & & & & & \\
\hline & & & & & & & & & & 0.39 & \\
\hline & & & & & & & & & & & \\
\hline
\end{tabular}

$=1.13, \mathrm{~s}=0.2713669$, skewness $=-0.79359$, kurtosis $=0.59954$. The boxplot of these observations displayed in Figure 3(a) indicates that the distribution is rightskewed. The TTT plot [39] of these data is shown in Figure 3(b). The TTT plot suggests an increasing failure rate and thus, the FW distribution could in principle be appropriate for modeling the current data. Table ?? provides the MLEs of the parameters and the values of $A^{*}, W^{*}, \mathrm{~K}-\mathrm{S}, \mathrm{AIC}, \mathrm{BIC}$, and loglikelihood for each model. On the basis of results listed in the table, we conclude that the FW distribution provides the best fit with the lowest values of model selection criteria. This indicates that the FW distribution has the ability to fit left-skewed data with increasing failure rate. For a visual comparison, we provide QQ-plots for all fitted models in Figure 4. Clearly, the FW model provides the closest fit to the data. 
TABLE 4. Simulation results for $\alpha=2, \lambda=3$.

\begin{tabular}{|c|c|c|c|c|c|c|c|c|c|c|c|}
\hline & Est. & MLE & LSE & WLS & $\mathrm{PCE}$ & MPS & MSADE & MSALDE & CVM & AD & RAD \\
\hline \multirow[t]{7}{*}{20} & $\operatorname{Bias}(\hat{\alpha})$ & $-2.474^{9}$ & $0.51^{4}$ & $0.51^{2}$ & $-2.904^{10}$ & $0.511^{7}$ & $0.51^{5}$ & $0.51^{6}$ & $0.51^{3}$ & $-1.360^{8}$ & $0.264^{1}$ \\
\hline & $\operatorname{RMSE}(\hat{\alpha})$ & $2.474^{9}$ & $0.51^{4}$ & $0.51^{2}$ & $2.904^{10}$ & $0.536^{7}$ & $0.51^{5}$ & $0.51^{6}$ & $0.51^{3}$ & $1.365^{8}$ & $0.364^{1}$ \\
\hline & $\operatorname{Bias}(\hat{\lambda})$ & $2.862^{8}$ & $0.01^{3}$ & $0.01^{5}$ & $85.223^{10}$ & $0.011^{6}$ & $0.01^{2}$ & $0.01^{1}$ & $0.01^{4}$ & $4.050^{9}$ & $-0.291^{7}$ \\
\hline & $\operatorname{RMSE}(\hat{\lambda})$ & $2.862^{8}$ & $0.01^{2}$ & $0.01^{4}$ & $114.487^{10}$ & $0.119^{6}$ & $0.01^{1}$ & $0.01^{5}$ & $0.01^{3}$ & $4.237^{9}$ & $0.536^{7}$ \\
\hline & $D_{\text {abs }}$ & $0.013^{7}$ & $0.00^{3}$ & $0.00^{1}$ & $1.000^{10}$ & $0.000^{6}$ & $0.00^{3}$ & $0.00^{5}$ & $0.00^{3}$ & $0.495^{9}$ & $0.146^{8}$ \\
\hline & $D_{\max }$ & $0.172^{7}$ & $0.00^{3}$ & $0.00^{1}$ & $1.000^{10}$ & $0.000^{6}$ & $0.00^{3}$ & $0.00^{5}$ & $0.00^{3}$ & $0.923^{9}$ & $0.219^{8}$ \\
\hline & $\sum \mathrm{Ranks}$ & $48^{8}$ & $19^{3}$ & $15^{1}$ & $60^{10}$ & $38^{7}$ & $19^{3}$ & $28^{5}$ & $19^{3}$ & $52^{9}$ & $32^{6}$ \\
\hline \multirow[t]{7}{*}{40} & $\operatorname{Bias}(\hat{\alpha})$ & $-2.473^{9}$ & $0.51^{6}$ & $0.51^{4}$ & $-2.91^{10}$ & $0.509^{3}$ & $0.51^{7}$ & $0.51^{8}$ & $0.51^{5}$ & $0.226^{1}$ & $0.235^{2}$ \\
\hline & $\operatorname{RMSE}(\hat{\alpha})$ & $2.474^{9}$ & $0.51^{5}$ & $0.51^{3}$ & $2.91^{10}$ & $0.512^{8}$ & $0.51^{6}$ & $0.51^{7}$ & $0.51^{4}$ & $0.274^{1}$ & $0.285^{2}$ \\
\hline & $\operatorname{Bias}(\hat{\lambda})$ & $2.862^{9}$ & $0.01^{3}$ & $0.01^{5}$ & $100.15^{10}$ & $0.014^{6}$ & $0.01^{2}$ & $0.01^{1}$ & $0.01^{4}$ & $-0.360^{8}$ & $-0.340^{7}$ \\
\hline & $\operatorname{RMSE}(\hat{\lambda})$ & $2.862^{9}$ & $0.01^{2}$ & $0.01^{4}$ & $129.73^{10}$ & $0.214^{6}$ & $0.01^{1}$ & $0.01^{5}$ & $0.01^{3}$ & $0.446^{7}$ & $0.450^{8}$ \\
\hline & $D_{\mathrm{abs}}$ & $0.013^{7}$ & $0.00^{3}$ & $0.00^{1}$ & $1.00^{10}$ & $0.000^{6}$ & $0.00^{3}$ & $0.00^{5}$ & $0.00^{3}$ & $0.145^{9}$ & $0.145^{8}$ \\
\hline & $D_{\max }$ & $0.260^{9}$ & $0.00^{3}$ & $0.00^{1}$ & $1.00^{10}$ & $0.000^{6}$ & $0.00^{3}$ & $0.00^{5}$ & $0.00^{3}$ & $0.212^{7}$ & $0.213^{8}$ \\
\hline & $\sum R$ anks & $52^{9}$ & $22^{3}$ & $18^{1}$ & $60^{10}$ & $35^{7.5}$ & $22^{3}$ & $31^{5}$ & $22^{3}$ & $33^{6.5}$ & $35^{7.5}$ \\
\hline \multirow[t]{7}{*}{60} & $\operatorname{Bias}(\hat{\alpha})$ & $-2.473^{9}$ & $0.51^{6}$ & $0.51^{4}$ & $-2.912^{10}$ & $0.509^{3}$ & $0.51^{7}$ & $0.51^{8}$ & $0.51^{5}$ & $0.460^{1}$ & $0.467^{2}$ \\
\hline & $\operatorname{RMSE}(\hat{\alpha})$ & $2.474^{9}$ & $0.51^{5}$ & $0.51^{3}$ & $2.912^{10}$ & $0.518^{8}$ & $0.51^{6}$ & $0.51^{7}$ & $0.51^{4}$ & $0.482^{1}$ & $0.489^{2}$ \\
\hline & $\operatorname{Bias}(\hat{\lambda})$ & $2.862^{9}$ & $0.01^{3}$ & $0.01^{5}$ & $106.919^{10}$ & $0.017^{6}$ & $0.01^{2}$ & $0.01^{1}$ & $0.01^{4}$ & $-0.710^{8}$ & $-0.700^{7}$ \\
\hline & $\operatorname{RMSE}(\hat{\lambda})$ & $2.862^{9}$ & $0.01^{2}$ & $0.01^{4}$ & $137.635^{10}$ & $0.303^{6}$ & $0.01^{1}$ & $0.01^{5}$ & $0.01^{3}$ & $0.730^{8}$ & $0.725^{7}$ \\
\hline & $D_{\mathrm{abs}}$ & $0.013^{7}$ & $0.00^{3}$ & $0.00^{1}$ & $1.000^{10}$ & $0.000^{6}$ & $0.00^{4}$ & $0.00^{5}$ & $0.00^{2}$ & $0.269^{9}$ & $0.269^{8}$ \\
\hline & $D_{\max }$ & $0.318^{7}$ & $0.00^{3}$ & $0.00^{1}$ & $1.000^{10}$ & $0.001^{6}$ & $0.00^{4}$ & $0.00^{5}$ & $0.00^{2}$ & $0.399^{8}$ & $0.400^{9}$ \\
\hline & $\sum \mathrm{Ranks}$ & $50^{9}$ & $22^{3}$ & $18^{1}$ & $60^{10}$ & $35^{7}$ & $24^{4}$ & $31^{5}$ & $20^{2}$ & $35^{7}$ & $35^{7}$ \\
\hline \multirow[t]{7}{*}{80} & $\operatorname{Bias}(\hat{\alpha})$ & $-2.473^{9}$ & $0.51^{6}$ & $0.510^{4}$ & $-2.913^{10}$ & $0.508^{3}$ & $0.51^{7}$ & $0.51^{8}$ & $0.51^{5}$ & $0.457^{1}$ & $0.463^{2}$ \\
\hline & $\operatorname{RMSE}(\hat{\alpha})$ & $2.474^{9}$ & $0.51^{4}$ & $0.511^{7}$ & $2.913^{10}$ & $0.516^{8}$ & $0.51^{5}$ & $0.51^{6}$ & $0.51^{3}$ & $0.473^{1}$ & $0.479^{2}$ \\
\hline & $\operatorname{Bias}(\hat{\lambda})$ & $2.862^{9}$ & $0.01^{3}$ & $0.011^{5}$ & $112.751^{10}$ & $0.019^{6}$ & $0.01^{2}$ & $0.01^{1}$ & $0.01^{4}$ & $-0.713^{8}$ & $-0.706^{7}$ \\
\hline & $\operatorname{RMSE}(\hat{\lambda})$ & $2.862^{9}$ & $0.01^{1}$ & $0.143^{5}$ & $144.653^{10}$ & $0.350^{6}$ & $0.01^{4}$ & $0.01^{3}$ & $0.01^{2}$ & $0.728^{8}$ & $0.724^{7}$ \\
\hline & $D_{\text {abs }}$ & $0.013^{7}$ & $0.00^{2}$ & $0.000^{5}$ & $1.000^{10}$ & $0.000^{6}$ & $0.00^{3}$ & $0.00^{4}$ & $0.00^{1}$ & $0.269^{9}$ & $0.269^{8}$ \\
\hline & $D_{\max }$ & $0.362^{7}$ & $0.00^{2}$ & $0.000^{5}$ & $1.000^{10}$ & $0.001^{6}$ & $0.00^{3}$ & $0.00^{4}$ & $0.00^{1}$ & $0.399^{8}$ & $0.399^{9}$ \\
\hline & $\sum$ Ranks & $50^{9}$ & $18^{2}$ & $31^{5}$ & $60^{10}$ & $35^{7}$ & $24^{3}$ & $26^{4}$ & $16^{1}$ & $35^{7}$ & $35^{7}$ \\
\hline \multirow[t]{7}{*}{100} & $\operatorname{Bias}(\hat{\alpha})$ & $-2.473^{9}$ & $0.51^{6}$ & $0.510^{4}$ & $-2.914^{10}$ & $0.508^{3}$ & $0.51^{7}$ & $0.51^{8}$ & $0.51^{5}$ & $0.456^{1}$ & $0.460^{2}$ \\
\hline & $\operatorname{RMSE}(\hat{\alpha})$ & $2.474^{9}$ & $0.51^{4}$ & $0.511^{7}$ & $2.914^{10}$ & $0.515^{8}$ & $0.51^{5}$ & $0.51^{6}$ & $0.51^{3}$ & $0.469^{1}$ & $0.473^{2}$ \\
\hline & $\operatorname{Bias}(\hat{\lambda})$ & $2.862^{9}$ & $0.01^{3}$ & $0.011^{5}$ & $115.576^{10}$ & $0.020^{6}$ & $0.01^{2}$ & $0.01^{1}$ & $0.01^{4}$ & $-0.715^{8}$ & $-0.709^{7}$ \\
\hline & $\operatorname{RMSE}(\hat{\lambda})$ & $2.862^{9}$ & $0.01^{1}$ & $0.142^{5}$ & $149.390^{10}$ & $0.372^{6}$ & $0.01^{4}$ & $0.01^{3}$ & $0.01^{2}$ & $0.727^{8}$ & $0.724^{7}$ \\
\hline & & $0.013^{7}$ & $0.00^{2}$ & $0.000^{5}$ & $1.000^{10}$ & $0.000^{6}$ & $0.00^{3}$ & $0.00^{4}$ & $0.00^{1}$ & $0.269^{9}$ & $0.269^{8}$ \\
\hline & $D_{\max }$ & $0.395^{7}$ & $0.00^{2}$ & $0.000^{5}$ & $1.000^{10}$ & $0.001^{6}$ & $0.00^{3}$ & $0.00^{4}$ & $0.00^{1}$ & $0.399^{8}$ & $0.399^{9}$ \\
\hline & $\sum$ Ranks & $50^{9}$ & & $31^{5}$ & $60^{10}$ & & $24^{3}$ & $26^{4}$ & $16^{1}$ & $35^{7}$ & $35^{7}$ \\
\hline
\end{tabular}

\section{Concluding Remarks}

This article studied the performance of different estimation methods for flexible Weibull distribution. The distribution parameters are estimated by eleven different methods of estimation, namely, the maximum likelihood estimators, least squares and weighted least squares estimators, the maximum product of spacings estimators, the minimum spacing absolute distance estimators, the minimum spacing absolute-log distance estimators, Cramér-von-Mises estimators, Anderson-Darling, right-tail Anderson-Darling, and the Bayes estimators. The results of the simulation study showed that among the frequentist estimators, Cramér-von-Mises estimators and Anderson-Darling perform better than their counterparts. Contrary to frequentist methods, Bayesian method outperformed the rest estimation methods. In the future, different estimation methods can be compared using censored and record data. Furthermore, different confidence intervals, like approximate, bootstrap, and 
TABle 5. Monte Carlo Markov Chain results for Bayesian analysis.

\begin{tabular}{|c|c|c|c|c|c|c|}
\hline Parametes & $n$ & Estimate & SD & MC error & $95 \%$ CI & Median \\
\hline \multirow[t]{5}{*}{$\alpha=0.5$} & 20 & 0.4990 & 0.5096 & 0.0051 & $(0.0125,1.912)$ & 0.3418 \\
\hline & 40 & 0.4995 & 0.5045 & 0.0035 & $(0.0122,1.872)$ & 0.3443 \\
\hline & 60 & 0.4992 & 0.4999 & 0.0029 & $(0.0124,1.865)$ & 0.3455 \\
\hline & 80 & 0.4998 & 0.4977 & 0.0023 & $(0.0128,1.845)$ & 0.346 \\
\hline & 100 & 0.4982 & 0.4950 & 0.0021 & $(0.0127,1.851)$ & 0.3452 \\
\hline \multirow[t]{5}{*}{$\lambda=0.5$} & 20 & 0.4968 & 0.5039 & 0.0051 & $(0.0119,1.866)$ & 0.3404 \\
\hline & 40 & 0.4976 & 0.4969 & 0.0033 & $(0.0128,1.86)$ & 0.3462 \\
\hline & 60 & 0.4969 & 0.4968 & 0.0026 & $(0.0121,1.845)$ & 0.3454 \\
\hline & 80 & 0.4964 & 0.4958 & 0.0023 & $(0.0122,1.841)$ & 0.3447 \\
\hline & 100 & 0.4965 & 0.4948 & 0.0022 & $(0.0122,1.837)$ & 0.3452 \\
\hline \multirow[t]{5}{*}{$\alpha=1.5$} & 20 & 1.489 & 0.8649 & 0.0089 & $(0.3095,3.635)$ & 1.318 \\
\hline & 40 & 1.488 & 0.855 & 0.0058 & $(0.3104,3.561)$ & 1.326 \\
\hline & 60 & 1.493 & 0.835 & 0.0051 & $(0.3065,3.559)$ & 1.325 \\
\hline & 80 & 1.497 & 0.8246 & 0.0043 & $(0.3091,3.563)$ & 1.326 \\
\hline & 100 & 1.498 & 0.8157 & 0.0038 & $(0.3078,3.58)$ & 1.33 \\
\hline \multirow[t]{5}{*}{$\lambda=0.5$} & 20 & 0.4934 & 0.4977 & 0.0046 & $(0.0132,1.836)$ & 0.3365 \\
\hline & 40 & 0.4976 & 0.4975 & 0.0034 & $(0.0123,1.845)$ & 0.3459 \\
\hline & 60 & 0.4991 & 0.4963 & 0.0028 & $(0.0119,1.838)$ & 0.3463 \\
\hline & 80 & 0.5008 & 0.4927 & 0.0027 & $(0.0127,1.847)$ & 0.3463 \\
\hline & 100 & 0.4996 & 0.4905 & 0.0023 & $(0.0127,1.846)$ & 0.3456 \\
\hline \multirow[t]{5}{*}{$\alpha=1.5$} & 20 & 1.501 & 0.8659 & 0.0093 & $(0.3163,3.629)$ & 1.336 \\
\hline & 40 & 1.501 & 0.8657 & 0.0065 & $(0.3155,3.611)$ & 1.335 \\
\hline & 60 & 1.497 & 0.8653 & 0.0050 & $(0.3118,3.606)$ & 1.335 \\
\hline & 80 & 1.498 & 0.8649 & 0.0046 & $(0.3125,3.607)$ & 1.334 \\
\hline & 100 & 1.499 & 0.8645 & 0.0039 & $(0.3119,3.604)$ & 1.331 \\
\hline \multirow[t]{5}{*}{$\lambda=2$} & 20 & 1.97 & 0.9931 & 0.0099 & $(0.5345,4.331)$ & 1.811 \\
\hline & 40 & 1.977 & 0.9874 & 0.0069 & $(0.5475,4.289)$ & 1.818 \\
\hline & 60 & 1.977 & 0.9822 & 0.0058 & $(0.5536,4.297)$ & 1.82 \\
\hline & 80 & 1.980 & 0.9820 & 0.0048 & $(0.5502,4.301)$ & 1.823 \\
\hline & 100 & 1.989 & 0.9814 & 0.0045 & $(0.5475,4.308)$ & 1.822 \\
\hline \multirow[t]{5}{*}{$\alpha=1.5$} & 20 & 2.982 & 1.222 & 0.0116 & $(1.098,5.859)$ & 2.813 \\
\hline & 40 & 2.979 & 1.220 & 0.0080 & $(1.103,5.849)$ & 2.807 \\
\hline & 60 & 2.976 & 1.218 & 0.0066 & $(1.101,5.842)$ & 2.806 \\
\hline & 80 & 2.99 & 1.188 & 0.0028 & $(1.093,5.816)$ & 2.822 \\
\hline & 100 & 2.989 & 1.176 & 0.0026 & $(1.094,5.813)$ & 2.822 \\
\hline \multirow[t]{5}{*}{$\lambda=2$} & 20 & 1.98 & 0.991 & 0.0099 & $(0.5403,4.333)$ & 1.816 \\
\hline & 40 & 1.986 & 0.9893 & 0.0075 & $(0.5383,4.324)$ & 1.825 \\
\hline & 60 & 1.988 & 0.9891 & 0.0056 & $(0.5407,4.331)$ & 1.829 \\
\hline & 80 & 1.989 & 0.9925 & 0.0028 & $(0.5408,4.361)$ & 1.824 \\
\hline & 100 & 1.989 & 0.9913 & 0.0024 & $(0.5377,4.368)$ & 1.824 \\
\hline
\end{tabular}

Bayesian can also be compared. Also, bias-corrected estimators can be studied for the flexible Weibull distribution.

\section{Conflict of interest}

On behalf of all authors, the corresponding author states that there is no conflict of interest. 


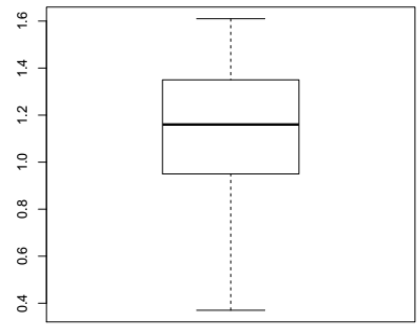

(a)

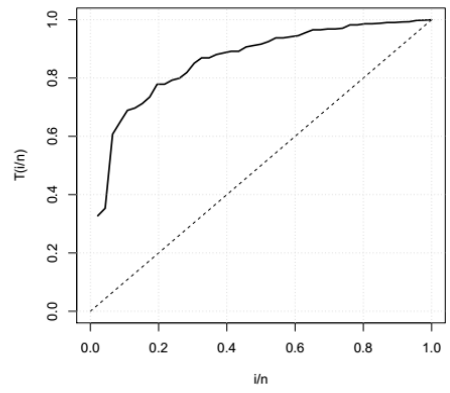

(b)

Figure 3. (a) Histogram (b) TTT plot for the strengths of glass fibres data.

TABLE 6. MLEs, their standard errors (in parentheses) and goodness-of-fit measures of the strengths of glass fibres data.

\begin{tabular}{|c|c|c|c|c|c|c|c|c|}
\hline Distribution & \multicolumn{2}{|c|}{ Estimates } & $A^{*}$ & $W^{*}$ & $\mathrm{~K}-\mathrm{S}$ & $\mathrm{AIC}$ & $\mathrm{BIC}$ & Loglikelihood \\
\hline $\mathrm{FW}(\alpha, \lambda)$ & 1.9908 & 2.96114 & 0.4157 & 0.0622 & 0.0605 & 10.6989 & 14.3562 & 3.34946 \\
\hline & $(0.2304)$ & $(0.3925)$ & & & & & & \\
\hline $\operatorname{Gamma}(\alpha, \theta)$ & $\begin{array}{l}11.6769 \\
(3.6130)\end{array}$ & $\begin{array}{c}0.0979 \\
(0.0313)\end{array}$ & 1.3219 & 0.1920 & 0.1324 & 26.3742 & 30.0315 & 11.1871 \\
\hline Weibull $(c, \lambda)$ & $\begin{array}{l}2.79206 \\
(0.2133)\end{array}$ & $\begin{array}{c}0.0490 \\
(0.0138)\end{array}$ & 0.5254 & 0.0661 & 0.0921 & 13.2132 & 16.8705 & 4.6065 \\
\hline $\log -\operatorname{normal}(\mu, \sigma)$ & $\begin{array}{c}0.0850 \\
(0.0437)\end{array}$ & $\begin{array}{c}0.2964 \\
(0.0309)\end{array}$ & 1.8996 & 0.2838 & 0.1596 & 30.5075 & 34.1648 & 13.2538 \\
\hline $\mathrm{NH}(\alpha, \lambda)$ & $\begin{array}{c}35.5990 \\
(27.5059)\end{array}$ & $\begin{array}{c}0.0193 \\
(0.0150)\end{array}$ & 0.8102 & 0.1129 & 0.4296 & 79.2266 & 82.8838 & 37.6133 \\
\hline $\operatorname{EE}(\alpha, \lambda)$ & $\begin{array}{l}20.4136 \\
(6.6018)\end{array}$ & $\begin{array}{c}3.1137 \\
(0.3384)\end{array}$ & 2.0367 & 0.3076 & 0.1601 & 33.2085 & 36.8658 & 14.6043 \\
\hline $\mathrm{BS}(\alpha, \beta)$ & $\begin{array}{c}0.3042 \\
(0.0317)\end{array}$ & $\begin{array}{c}1.0797 \\
(0.0478)\end{array}$ & 2.0263 & 0.3029 & 0.1714 & 31.9066 & 35.5639 & 13.9533 \\
\hline $\mathrm{IG}(\mu, \lambda)$ & $\begin{array}{c}1.1312 \\
(0.0516)\end{array}$ & $\begin{array}{c}311.8473 \\
(2.4703)\end{array}$ & 2.0538 & 0.3075 & 0.1712 & 32.2376 & 35.8949 & 14.1188 \\
\hline
\end{tabular}

\section{REFERENCES}

[1] M. Bebbington, C.-D. Lai, R. Zitikis, A flexible weibull extension, Reliability Engineering \& System Safety 92 (6) (2007) $719-726$.

[2] K. Vijay, L. Uwe, reliaR: Package for some probability distributions, https://CRAN. R-project.org/package=reliaR https://CRAN.R-project.org/package=reliaR 0th Edition (2011).

[3] S. K. Singh, U. Singh, V. K. Sharma, Bayesian estimation and prediction for flexible Weibull model under type-II censoring scheme, Journal of Probability and Statistics 2013 (2013) 146140. doi:https://doi.org/10.1155/2013/146140 

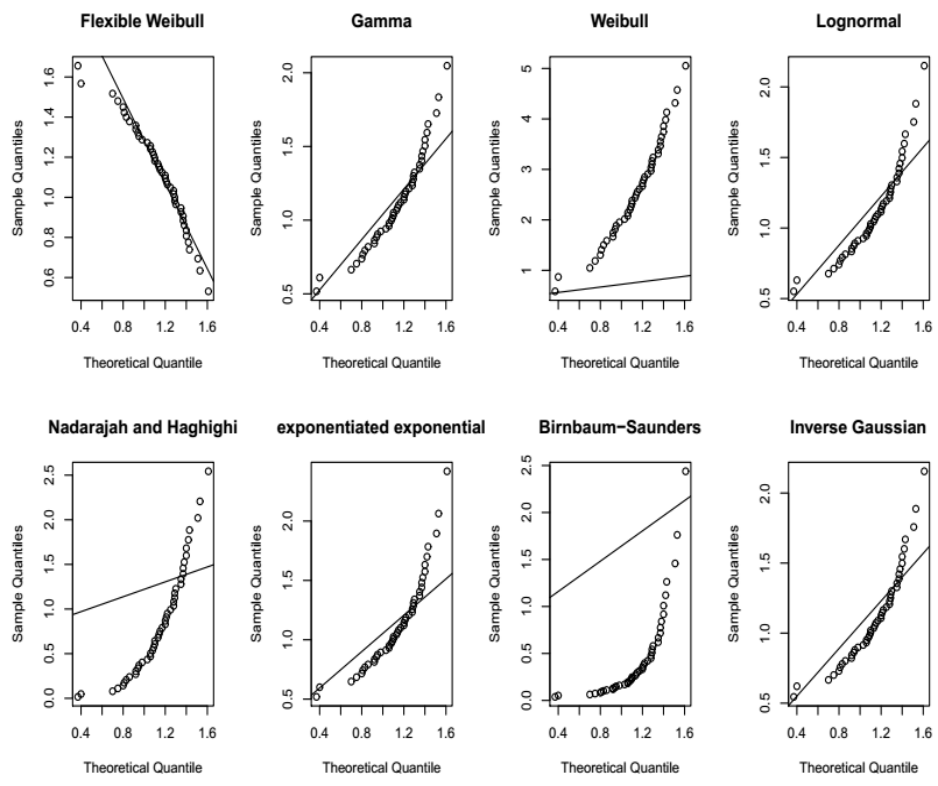

Figure 4. Q-Q plots for the strengths of glass fibre data.

[4] A. Nasr, S. Gasmi, F. B. Hmida, Parameter estimation of the flexible Weibull distribution for type I censored samples, Journal of Applied Statistics 44 (14) (2017) 2499-2512. doi: $10.1080 / 02664763.2016 .1257588$

[5] A. Mustafa, B. S. El-Desouky, S. AL-Garash, Odd generalized exponential flexible Weibull extension distribution, Journal of Statistical Theory and Applications 17 (2018) 77-90. doi: https://doi.org/10.2991/jsta.2018.17.1.6

[6] F. Prataviera, E. M. Ortega, G. M. Cordeiro, R. R. Pescim, B. Verssani, A new generalized odd log-logistic flexible Weibull regression model with applications in repairable systems, Reliability Engineering \& System Safety 176 (2018) 13 - 26. doi:https://doi.org/10.1016/ j.ress.2018.03.034

[7] S. Park, J. Park, A general class of flexible Weibull distributions, Communications in Statistics - Theory and Methods 47 (4) (2018) 767-778. doi:10.1080/03610926.2015.1118509

[8] V. Sharma, Estimation and prediction for type-II hybrid censored data follow flexible Weibull distribution, Statistica 77 (4) (2018) 385-414. doi:10.6092/issn.1973-2201/6779

[9] V. M. C. Lima, F. Cribari-Neto, Penalized maximum likelihood estimation in the modified extended Weibull distribution, Communications in Statistics - Simulation and Computation 48 (2) (2019) 334-349. doi:10.1080/03610918.2017.1381735

[10] P. G. Sankaran, K. M. Dileep, Reliability properties of proportional hazards relevation transform, Metrika 82 (4) (2019) 441-456. doi:10.1007/s00184-018-0681-0

[11] O. M. Bdair, R. R. A. Awwad, G. K. Abufoudeh, M. F. M. Naser, Estimation and prediction for flexible Weibull distribution based on progressive type II censored data, Communications in Mathematics and Statistics (2019) 1-2:doi:https://doi.org/10.1007/ s40304-018-00173-0 
[12] A. E.-B. A. Ahmad, M. Ghazal, Exponentiated additive weibull distribution, Reliability Engineering \& System Safety 193 (2020) 106663. doi:https://doi.org/10.1016/j.ress. 2019.106663

[13] S. Dey, T. Dey, D. Kundu, Two-parameter Rayleigh distribution: Different methods of estimation, American Journal of Mathematical and Management Sciences 33 (1) (2014) 55-74.

[14] S. Dey, S. Ali, C. Park, Weighted exponential distribution: properties and different methods of estimation, Journal of Statistical Computation and Simulation 85 (18) (2015) 3641-3661.

[15] S. Dey, T. Dey, S. Ali, M. S. Mulekar, Two-parameter Maxwell distribution: Properties and different methods of estimation, Journal of Statistical Theory and Practice 10 (2) (2016) 291-310.

[16] S. Dey, D. Kumar, P. L. Ramos, F. Louzada, Exponentiated Chen distribution: Properties and estimation, Communications in Statistics - Simulation and Computation 46 (10) (2017) $8118-8139$.

[17] S. Dey, B. Al-Zahrani, S. Basloom, Dagum distribution: Properties and different methods of estimation, International Journal of Statistics and Probability 6 (2) (2017) 74-92.

[18] S. Dey, E. Raheem, S. Mukherjee, Statistical Properties and Different Methods of Estimation of Transmuted Rayleigh Distribution, Revista Colombiana de EstadÃstica 40 (2017) 165 203.

[19] S. Dey, E. Raheem, S. Mukherjee, H. K. T. Ng, Two parameter exponentiated Gumbel distribution: properties and estimation with flood data example, Journal of Statistics and Management Systems 20 (2) (2017) 197-233.

[20] S. Dey, C. Zhang, A. Asgharzadeh, M. Ghorbannezhad, Comparisons of methods of estimation for the NH distribution, Annals of Data Science 4 (4) (2017) 441-455.

[21] J. Kenney, E. Keeping, Mathematics of statistics, no. v. 2 in Mathematics of Statistics, Princeton: Van Nostrand, 1962.

[22] J. J. A. Moors, A quantile alternative for kurtosis, Journal of the Royal Statistical Society. Series D (The Statistician) 37 (1) (1988) 25-32.

[23] M. Shaked, J. Shanthikumar, Stochastic Orders, Springer New York, 2007.

[24] J. J. Swain, S. Venkatraman, J. R. Wilson, Least-squares estimation of distribution functions in Johnson's translation system, Journal of Statistical Computation and Simulation 29 (4) (1988) 271-297.

[25] J. H. K. Kao, Computer methods for estimating Weibull parameters in reliability studies, IRE Transactions on Reliability and Quality Control PGRQC-13 (1958) 15-22.

[26] J. H. K. Kao, A graphical estimation of mixed Weibull parameters in life-testing of electron tubes, Technometrics 1 (4) (1959) 389-407.

[27] N. Mann, R. Schafer, N. Singpurwalla, Methods for statistical analysis of reliability and life data, Wiley, 1974.

[28] R. C. H. Cheng, N. A. K. Amin, Maximum product of spacings estimation with application to the lognormal distribution, Tech. rep. (1979).

[29] R. C. H. Cheng, N. A. K. Amin, Estimating parameters in continuous univariate distributions with a shifted origin, Journal of the Royal Statistical Society. Series B (Methodological) 45 (3) (1983) 394-403.

[30] B. Ranneby, The maximum spacing method. an estimation method related to the maximum likelihood method, Scandinavian Journal of Statistics 11 (2) (1984) 93-112.

[31] P. D. M. MacDonald, Comment on "an estimation procedure for mixtures of distributions" by Choi and Bulgren, Journal of the Royal Statistical Society. Series B (Methodological) 33 (2) (1971) 326-329.

[32] T. W. Anderson, D. A. Darling, Asymptotic theory of certain goodness of fit criteria based on stochastic processes, The Annals of Mathematical Statistics 23 (2) (1952) 193-212.

[33] T. W. Anderson, D. A. Darling, A test of goodness of fit, Journal of the American Statistical Association 49 (268) (1954) 765-769. 
[34] A. N. Pettitt, A two-sample Anderson-Darling rank statistic, Biometrika 63 (1) (1976) 161168.

[35] M. A. Stephens, EDF statistics for goodness of fit and some comparisons, Journal of the American Statistical Association 69 (347) (1974) 730-737.

[36] L. Devroye, Non-Uniform Random Variate Generation, Springer-Verlag, 1986.

[37] S. Nadarajah, F. Haghighi, An extension of the exponential distribution, Statistics 45 (6) (2011) 543-558.

[38] R. L. Smith, J. C. Naylor, A comparison of maximum likelihood and bayesian estimators for the three- parameter weibull distribution, Journal of the Royal Statistical Society. Series C (Applied Statistics) 36 (3) (1987) 358-369.

[39] M. Aarset, How to identify a bathtub hazard rate, IEEE Transactions on Reliability R-36 (1) (1987) 106-108.

Current address: Sajid Ali: Department of Statistics, Quaid-i-Azam University, Islamabad, Pakistan

E-mail address: sajidali.qau@hotmail.com

ORCID Address: http://orcid.org/0000-0003-4868-7932

Current address: Sanku Dey: Department of Statistics, St. Anthonys College, Shillong, India E-mail address: sankud66@gmail.com;

ORCID Address: http://orcid.org/0000-0002-2157-3997

Current address: M. H. Tahir: Department of Statistics, The Islamia University of Bahawalpur, Bahawalpur, Pakistan

E-mail address: mtahir.stat@gmail.com

ORCID Address: http://orcid.org/0000-0003-0024-0322

Current address: Muhammad Mansoor: Department of Statistics, Government Sadiq Egerton College, Bahawalpur, Pakistan

E-mail address: mansoor.abbasi143@gmail.com.

ORCID Address: http://orcid.org/0000-0002-6900-8233 